\title{
Design of the Prevention of Adult Caries Study (PACS): A randomized clinical trial assessing the effect of a chlorhexidine dental coating for the prevention of adult caries
}

William M Vollmer ${ }^{1 *}$, Athena S Papas², James D Bader ${ }^{3}$, Gerardo Maupomé ${ }^{4}$, Christina M Gullion', Jack F Hollis ${ }^{1}$, John J Snyder ${ }^{5}$, Jeffrey L Fellows ${ }^{1}$, Reesa L Laws ${ }^{1}$, B Alexander White ${ }^{6}$, the PACS Collaborative Research Group

\begin{abstract}
Background: Dental caries is one of the primary causes of tooth loss among adults. It is estimated to affect a majority of Americans aged 55 and older, with a disproportionately higher burden in disadvantaged populations. Although a number of treatments are currently in use for caries prevention in adults, evidence for their efficacy and effectiveness is limited.

Methods/Design: The Prevention of Adult Caries Study (PACS) is a multicenter, placebo-controlled, double-blind, randomized clinical trial of the efficacy of a chlorhexidine $(10 \% \mathrm{~W} / \mathrm{V})$ dental coating in preventing adult caries. Participants ( $n=983$ ) were recruited from four different dental delivery systems serving four diverse communities, including one American Indian population, and were randomized to receive either chlorhexidine or a placebo treatment. The primary outcome is the net caries increment (including non-cavitated lesions) from baseline to 13 months of follow-up. A cost-effectiveness analysis also will be considered.
\end{abstract}

Discussion: This new dental treatment, if efficacious and approved for use by the Food and Drug Administration (FDA), would become a new in-office, anti-microbial agent for the prevention of adult caries in the United States.

Trial Registration Number: NCT00357877.

\section{Background}

Although children are the primary recipients of cariesprevention programs in the United States (US), a recent meta-analysis concluded that older adults experience caries at the same or even higher rates [1]. Indeed, dental caries is one of the primary causes of tooth loss among adults [2-4], and is estimated to affect a majority of Americans aged 55 and older [5]. Root caries in particular is one of the most common chronic infectious diseases of midlife. Among Americans aged 45-64, root caries is more prevalent (at 35\%) [6] than chronic joint symptoms (32\%), hypertension (32\%), arthritis (29\%), symptoms of mental illness (14\%), heart disease (13\%),

\footnotetext{
* Correspondence: william.vollmer@kpchr.org

'Center for Health Research, 3800 N. Interstate Blvd, Portland, Oregon 97227, USA

Full list of author information is available at the end of the article
}

and diabetes (10\%) [7]. Data from a largely employed population with dental insurance found that mean annual spending on adult dental care peaks between ages 55 and 64 , at about $\$ 700$ per capita, and that caries-related treatment accounts for about half of these expenditures [8].

Adult caries can be particularly devastating in disadvantaged populations, such as the uninsured and certain racial and ethnic minority groups [5]. One subgroup that is particularly affected by dental caries is American Indians. Based on data from the Indian Health Service's 1999 oral health survey [9], 68\% of 19-year-old American Indians have experienced caries, compared to $24 \%$ of non-American-Indian 15-19-year-olds. Currently only about half of Americans (and just 1 in 5 over age 65) have dental insurance $[10,11]$. While the literature on the impact of dental insurance on dental health is
C Biomed Central

C 2010 Vollmer et al; licensee BioMed Central Ltd. This is an Open Access article distributed under the terms of the Creative Commons Attribution License (http://creativecommons.org/licenses/by/2.0), which permits unrestricted use, distribution, and reproduction in any medium, provided the original work is properly cited. 
limited, it is clear that the uninsured have a higher level of edentulism (27\% vs. $18.3 \%$ among insured) [12], a higher number of untreated caries [13], and a lower likelihood of visiting the dentist than the insured $[14,15]$. When they do see the dentist, the uninsured are more likely to receive a filling or extraction, or other acute care, and are somewhat less likely to receive preventive services.

Although several practices are recommended to prevent adult caries, a 2001 National Institutes of Health (NIH) consensus panel concluded that the evidence base for adult caries prevention is limited [16]. Everyone should be advised to floss daily and use fluoridated toothpaste 2-3 times per day. Adjunctive treatments may be used, including in-office fluoride gels, professionally applied fluoride varnish, or home use of prescription $(5000 \mathrm{ppm})$ topical fluoride [17-19].

Chlorhexidine is an antimicrobial that may be an effective adjunctive treatment for caries prevention. To date, chlorhexidine rinse $(0.12 \% \mathrm{w} / \mathrm{v})$ has been approved for use to reduce gingivitis, but not for caries prevention. Chlorhexidine rinse, when used in an alternating sequence of daily use for one month, then weekly for 5 months, failed to reduce caries more than a placebo (quinidine) rinse over 5 years in a randomized study of 1101 adults 65 years and older [20]. In addition, continual use of chlorhexidine rinse may stain teeth.

Two placebo-controlled trials of the efficacy of a chlorhexidine $(10 \% \mathrm{w} / \mathrm{v})$ dental coating applied by a dental hygienist or dentist to prevent caries were reported in 2000 [21,22]. Possible therapeutic advantages of this formulation include a much higher chlorhexidine concentration and longer contact time with the tooth surface, which should intensify the anti-cariogenic effects of chlorhexidine. In addition, in-office application avoids patient compliance issues.

The first study enrolled 240 adults with xerostomia aged 40-80 with an elevated risk of dental caries [21]. Participants were randomly assigned to active treatment or to one of two control conditions (either a sham treatment that contained quinine hydrochloride to mimic the bitter taste of chlorhexidine or a placebo control) and followed for 13 months, with four initial weekly treatments followed by a fifth treatment at six months. Relative to placebo, the active treatment group had a $24.5 \%$ greater reduction in combined root and coronal caries increment ( $p=0.03)$, a $40.8 \%$ reduction in root caries increment $(\mathrm{p}=0.02)$, and a $14.4 \%$ reduction in coronal caries increment $(p=0.06)$. Although caries increment scores also were reduced for the active treatment relative to the sham groups $(13.8 \%, 32.5 \%$, and $2.2 \%)$, these reductions were not statistically significant (potentially because of the antimicrobial properties of the sham agent, quinine hydrochloride).
The second study enrolled 1,240 economically disadvantaged adolescents aged 11-13 years with a history of decay and an elevated level of Streptococcus mutans $(250,000 \mathrm{cfu} / \mathrm{mL})$. Participants were randomly assigned to one of four arms: active drug, a placebo coating plus best practice preventive care, a best practice preventive care control condition, and a usual care control condition. All participants were followed for three years. Participants in active and placebo-coating arms received four initial weekly treatments, followed by additional treatments at 12 and 24 months post- randomization. Participants also received up to two additional treatments each year, depending upon their level of salivary S. mutans. An intent-to-treat analysis found no treatment effect overall [22], but adherence to the study treatment was poor, possibly affecting the results. Unpublished secondary analysis in the per-protocol sample found significant reduction in caries among girls in the active treatment group compared to girls in control arms, as well as a gender difference in use of sugary carbonated drinks, which may have reduced efficacy.

The current manuscript describes the protocol for the Prevention of Adult Caries Study (PACS), which represents a third evaluation of the chlorhexidine dental coating and is being conducted as a "pivotal" study under US Food and Drug Administration IND \#45,466. PACS is a randomized clinical trial being conducted over a 13-month observation period among adult participants from four diverse communities in the United States, each of which is served by distinctly different dentalcare delivery systems. Of note, PACS includes a large uninsured population lacking regular access to dental care, the western Navajo Nation and Hopi populations in northern Arizona, and a large metropolitan community without a fluoridated water supply. The primary objectives of PACS are (1) to test the hypothesis that the chlorhexidine dental coating, compared to a placebo coating, will reduce dental caries increment in at-risk adults from baseline to the 13-month follow-up visit; and (2) to evaluate the cost and cost-effectiveness of using the treatment from patient, program, and provider perspectives.

\section{Methods/Design}

PACS is a multicenter, placebo-controlled, doubleblind, randomized clinical trial. Participating institutions include the Kaiser Permanente Center for Health Research in Portland, OR, which serves as the data coordinating center, and four clinical centers: Tufts University School of Dental Medicine (TUSDM) in Boston, MA; the Tuba City Regional Health Care Corporation (TCRHCC) on the Navajo Reservation in northern Arizona; the Dental Service of Massachusetts clinic in Southborough, MA (a part of Delta Dental of 
Massachusetts); and the Kaiser Permanente Dental Care Program and Permanente Dental Associates in Portland, OR. The study chair is located at Tufts University, and this center also is the location of the microbiology laboratory. The study is sponsored by the National Institute of Dental and Craniofacial Research (NIDCR). The product manufacturer, CHX Technologies Inc., provides the study treatments, a Good Clinical Practice (GCP) auditor (Schiff \& Associates), and some additional funding and quality assurance of the product. Independent oversight of trial activities is provided by a Data and Safety Monitoring Board appointed by the NIDCR. The study was approved by the Institutional Review Boards of each participating institution and by the FDA, and all participants provided written informed consent.

\section{Study Population}

The study population consists of individuals aged 1880 years, having 20 or more intact teeth and deemed at increased risk of dental caries due to the presence of one or more cavitated lesions at screening (Table 1). We excluded participants for whom the study treatment would be contraindicated, as well as participants with health conditions that might affect the measurement of study outcomes or their ability to successfully complete the study. We targeted four communities with varying fluoride exposure, dental reimbursement, and overall risk of caries (Table 2). Financial incentives varied by site and included cash incentives, reimbursement of travel expenses and, for some individuals, reimbursement for the cost of required restorative care. Recruitment began in February, 2007 and concluded in August, 2008.

All participants attended an initial screening visit to assess eligibility. Eligible participants then received necessary restorative care for all cavitated lesions prior to returning for a randomization visit, at which time additional baseline measurements (including a baseline caries examination) were obtained. Individuals who still had cavitated lesions at this point were referred back to their treating dentist for further restorative care prior to randomization, and the randomization visit was then repeated.

Eligible participants were randomized to receive five applications of either a chlorhexidine $(10 \% \mathrm{w} / \mathrm{v})$ or placebo dental coating over a seven-month interval. A computerized randomization process confirmed participant eligibility prior to issuing randomization assignments. The latter were stratified by clinical center and age and, within each strata, clustered in blocks of varying sizes. Participants and staff were blinded to treatment assignment. Overall, we randomized 983 participants, close to our goal of 1000 .

Table 1 Eligibility Criteria for PACS

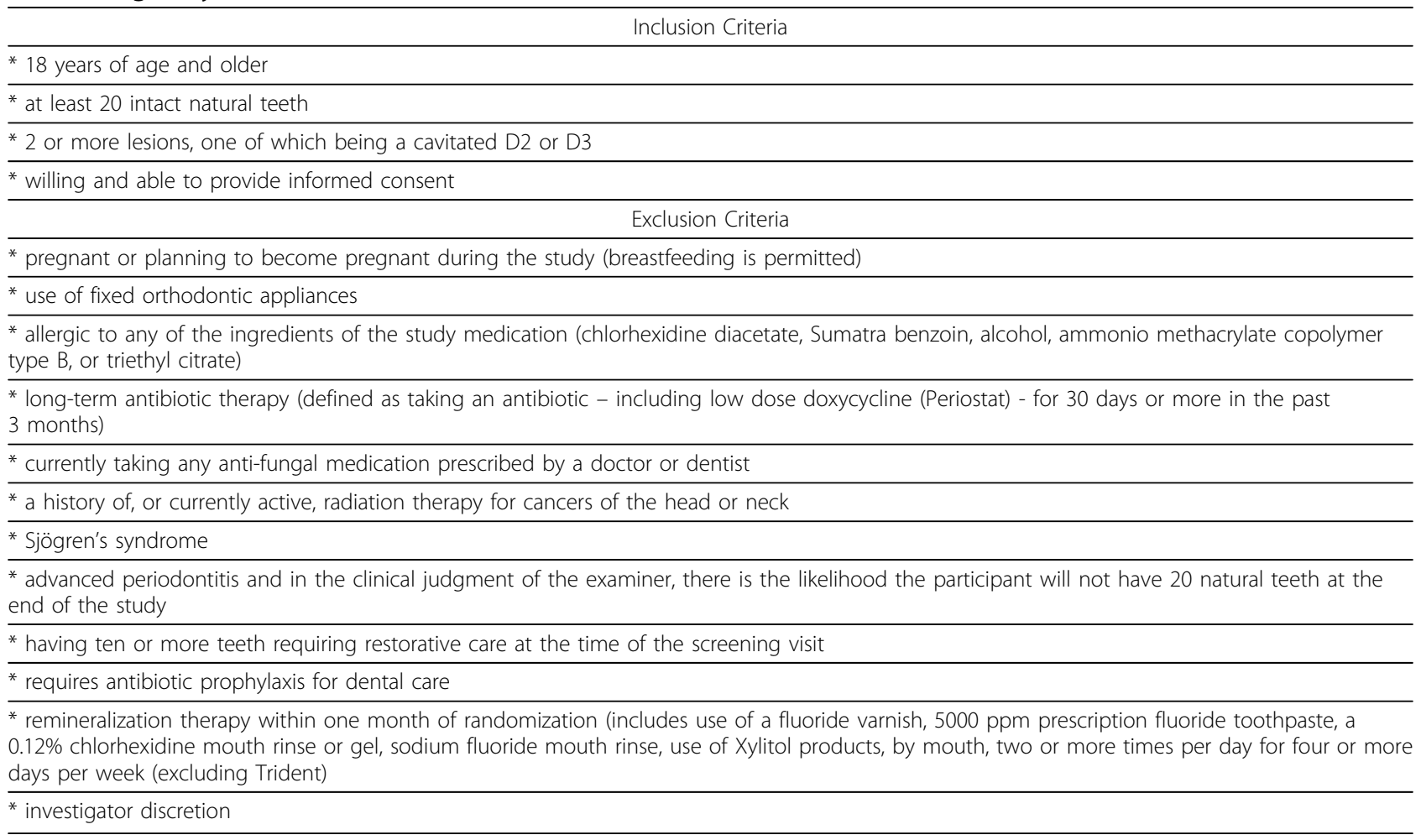


Table 2 Characteristics of PACS Clinical Center Populations

\begin{tabular}{lcccc}
\hline Study Center & Recr quota & Fluoride in water & Dental reimbursement & Est. caries prevalence versus US average \\
\hline KP Portland & 400 & Partly & Pre-paid managed care & Same \\
\hline Tufts & 200 & Yes & Majority uninsured & Same \\
\hline Dental Service of MA, Southborough & 200 & Partly & Primarily prepaid fee-for-service & Same \\
\hline Tuba City & 200 & Partly & Access to free care & Higher \\
\hline
\end{tabular}

\section{Schedule of Activities}

Treatment and data collection occurred at seven visits during the study period (Table 3 ). We collected baseline data and determined study eligibility at both the screening visit (SV) and randomization (V1) visits, which had to occur within 4 months of each other. The first treatment application occurred immediately following randomization at the V1 visit. Three additional treatment applications took place on a roughly weekly basis at the second through fourth visits (V2- V4), followed by a fifth and final treatment application seven months post- randomization as part of the fifth visit (V5). The V5 visit also included a comprehensive outcome assessment, together with a dental caries examination. Active caries discovered at the V5 visit had to be restored before the final treatment application. The final datacollection visit (V6) was scheduled six months later, or approximately 13 months post-randomization. This visit included a comprehensive outcome assessment.

Participants at the Tufts clinical center also were asked to take part in an optional substudy to assess the effect of the study medication on resistant (S. mutans)

Table 3 Schedule of Study Visits

\begin{tabular}{|c|c|c|c|c|c|c|c|c|c|}
\hline & SV $\left(t_{s}\right)$ & V1 $\left(t_{0}\right)$ & $\mathrm{V} 2\left(\mathrm{t}_{1}\right)$ & V3 $\left(t_{2}\right)$ & V4 $\left(t_{3}\right)$ & V5 $\left(t_{4}\right)$ & $\begin{array}{c}\text { V5a }\left(t_{5}\right) \\
\text { Tufts only }\end{array}$ & V6 $\left(t_{6}\right)$ & $\begin{array}{l}\text { V6a }\left(t_{7}\right) \\
\text { Tufts only }\end{array}$ \\
\hline Target date & & $t_{s}+4 \operatorname{mos}$ & $t_{0}+7 d$ & $t_{1}+7 d$ & $t_{2}+7 d$ & $\mathrm{t}_{0}+7 \mathrm{mos}$ & $t_{0}+10 \mathrm{mos}$ & $t_{0}+13 \mathrm{mos}$ & $t_{0}+19 \operatorname{mos}$ \\
\hline Allowable window & & & 5-14d & 5-14d & $5-14 d$ & $\pm 1 \mathrm{mo}$ & $\pm 1 \mathrm{mo}$ & $\pm 1 \mathrm{mo}$ & $\pm 1 \mathrm{mo}$ \\
\hline Eligibility questions & $x$ & $x^{1}$ & & & & & & & \\
\hline Exam for eligibility & $x$ & & & & & & & & \\
\hline Demographic questions & $x$ & & & & & & & & \\
\hline Informed consent & $x$ & $x^{2}$ & & & & & & & \\
\hline Dental restorations & $x^{3}$ & & & & & $x^{3}$ & & $x^{3}$ & \\
\hline Pregnancy test & $x$ & $x$ & & & & $x$ & & & \\
\hline Acidic drink consumption questions & $x$ & & & & $x$ & $x$ & & $x$ & \\
\hline Medical HX questions & & $x$ & & & & $x$ & & $x$ & \\
\hline Oral Health HX questions & & $x$ & & & & & & $x$ & \\
\hline Randomization & & $x$ & & & & & & & \\
\hline Tooth Surface Exam & & $x$ & & & & $x$ & & $x$ & \\
\hline Coating Application & & $x$ & $x$ & $x$ & $x$ & $x$ & & & \\
\hline Adverse Events questions & & $x$ & $x$ & $x$ & $x$ & $x$ & $x$ & $x$ & $x$ \\
\hline Medication questions & $x$ & $x$ & $x$ & $x$ & $x$ & $x$ & $x$ & $x$ & $x$ \\
\hline Soft Tissue Exam & & $x$ & & & & $x$ & & $x$ & \\
\hline Travel time/costs & & $x$ & & & & $x$ & & $x$ & \\
\hline Care Utilization & & & & & & & & $x$ & \\
\hline Candidiasis Lab Tracking & $x$ & $x$ & $x$ & $x$ & $x$ & $x$ & $x$ & $X$ & $X$ \\
\hline Medication Price Survey & & $X$ & & & & & & & \\
\hline $\begin{array}{l}\text { Microbiology } \\
\text { Tufts only }\end{array}$ & & $x^{4}$ & & & & $X^{4}$ & $X^{4}$ & $x^{5}$ & $X^{5}$ \\
\hline \multicolumn{10}{|c|}{ 15ome eligibility information will be reassessed at V1. } \\
\hline \multicolumn{10}{|c|}{ ªlthough not required, some study centers will use separate screening and randomization consent forms. } \\
\hline \multirow{2}{*}{\multicolumn{10}{|c|}{$\begin{array}{l}{ }^{3} \text { following the screening visit (SV), any needed dental restorations are made prior to randomization (V1); additional restorative work, if needed, is performed } \\
\text { following the dental examinations at the V } 5 \text { and V6 visits. } \\
{ }^{4} \text { testing for presence and level of S. mutans and C. albicans in a subset of patients at the Tufts clinical center. }\end{array}$}} \\
\hline & & & & & & & & & \\
\hline \multicolumn{10}{|c|}{${ }^{5}$ additional testing for S. mutans and C. albicans may occur at V6 and V6a depending on results of earlier tests. } \\
\hline
\end{tabular}


and opportunistic (Candida albicans) infections in the oral cavity. At each of the V1, V5, V5a, V6, and V6a visits, selected participants provided a sample of stimulated whole saliva for analyses of $S$. mutans sensitivity to chlorhexidine and swabs of the right and left buccal cheek mucosa for analyses of levels of C. albicans. Specimens were processed and analyzed by staff trained in Good Laboratory Practice (GLP) at the microbiology laboratory, which forwarded the results directly to the data coordinating center. A total of 143 Tufts subjects participated in this substudy.

\section{Study Treatments}

Study treatments were applied in-office by a dental hygienist after a brief rubber cup prophylaxis with nonfluoridated paste and scaling as needed. The coatings were applied in two stages. In stage 1, the first coating was applied to all tooth surfaces in a given quadrant of the dentition. This was immediately followed by application of the second coating (labeled Stage 2 on the vials) to the same quadrant. The other three quadrants were similarly treated and the coatings were air dried to ensure proper hardening. All dental hygienists were trained and certified in the proper application of the coatings, and the entire process typically took $20 \mathrm{~min}$ utes to complete.

The stage 1 coating contains either chlorhexidine diacetate suspended in a solution of Sumatra benzoin and alcohol (the active treatment) or simply the Sumatra benzoin and alcohol solution (the placebo treatment). The second coating is a proprietary aqueous dispersion of inert methacrylate approved for use by the FDA under license K013671. This second coating lasts until it is abraded by hard foods or is brushed from the teeth, and is designed to give the chlorhexidine extended contact time on the enamel.

The component medications used in both the Stage 1 and Stage 2 coatings were made under Good Manufacturing Practices[23] in an FDA-approved facility. All treatments were packaged in a cardboard box containing ten 2 -mL capped amber glass vials (sufficient for all 5 study treatments). Each vial contained either $1 \mathrm{~mL}$ of the Stage 1 coating or $1 \mathrm{~mL}$ of the Stage 2 coating. The box was labeled for PACS and had a tamper-proof seal. Stage 1 and Stage 2 vials had different colored labels, and each vial was labeled for one of the five visits. The treatment boxes were shipped overnight in refrigerated packaging from the study pharmacist to the clinical centers; thermometers measured trans-shipment temperatures. Upon receipt by the clinical centers, the treatment boxes were immediately refrigerated. Studies have demonstrated that the study treatments are stable at room temperature for longer than three months and at refrigerated temperatures for 30 months [24,25].
For participants in the active treatment arm, the mean dose of chlorhexidine at each application visit was expected to be approximately $33 \mathrm{mg}$ (or $330 \mu \mathrm{L}$ ), so that the cumulative mean dose for participants completing all five applications was expected to be $165 \mathrm{mg}$.

\section{Blinding of Study Treatments}

Active and placebo-coating materials were packaged identically and were distinguishable only by a numerical label. Each box of treatment material contained a complete supply of coatings for one person for the entire study. Individual vials, together with the box in which they were packaged, were affixed with the same label. The sequence of numerical codes was random with respect to likelihood that the package contained active or placebo coating. Packing was done by a central facility under contract to $\mathrm{CHX}$ Technologies Inc., and active and control boxes were prepared in separate production runs using label identifications (IDs) supplied by the data coordinating center. Independent verification of a random sample of boxes was done to confirm that the correct labels were assigned to the active and placebo boxes.

Since prior studies have shown that differences in taste or appearance between the study medication and placebo were imperceptible when substances were applied according to protocol, the likelihood of inadvertent unblinding of the patient, dental team, or other clinic staff is minimal. In the unlikely event that staff do become unblinded to the treatment status of a given participant, the nature of the treatment allocation process (as described above) means that the unblinding would be a single event and that nothing could be inferred about other participants' treatment assignments.

\section{Caries Examination and PACS Taxonomy}

Caries were diagnosed visually by calibrated examiners using a Community Periodontal Index of Treatment Needs (CPITN) probe, an unblemished, non-magnifying plane mirror; and standard dental operating light and chair. Use of loupes was according to local practice. The participants' teeth were dried for five seconds with an air/water syringe to better enable the examination of tooth surfaces. At the request of the FDA, radiographs were not used in diagnosis. Each central, lateral, and cuspid tooth was deemed to have five coronal (including the incisal) surfaces and four root surfaces. Examiners made only one diagnostic judgment per tooth surface.

Two initial 4-day training and calibration sessions were held at the two East Coast and West Coast sites with a gold standard examiner. These were followed by three recalibrations at roughly nine-month intervals.

The PACS taxonomy of adult dental caries used the nomenclature of Pitts and Fyffe [26] that identifies 
three basic stages of lesions: non-cavitated lesions (D1); lesions where the cavitation extends into, but not through, the enamel (D2); and cavitated lesions that involve the dentine (D3). (A modified taxonomy that does not include the D3 score is used for root surfaces.) Each stage of decay involves different and distinct treatment implications in terms of cost, staff time, and management. The D1, D2, and D3 designations are well understood by the community dentists in all four PACS clinical centers. This taxonomy has been used by Banting et al [21]. Papas et al.[27] and Chesters et al. [28] to detect caries incidence. The descriptors for the D1, D2, and D3 classifications in PACS are adapted from the International Caries Detection and Assessment System (ICDAS) II [29], as shown in Table 4. ICDAS II established objective clinical signs that have been associated with severity levels of dental caries (particularly the non-cavitated lesion) and verified histologically, and uses a 2-digit numeric code to classify each tooth site. The PACS taxonomy does not make a number of distinctions that are in the ICDAS II taxonomy. Instead, we have collapsed codes as follows:

- The first digit codes $0,1,2$ are collapsed into $S$ (sound or sealed surface), 3, 4, 7, 8 are collapsed into $F$ (filled), and 5, 6 are collapsed into $C$ (crowned)

- The second digit codes 1 and 2 were collapsed into a single D1 code (uncavitated lesion). This was done in recognition of the fact that it is difficult to train dentists in detecting pre-clinical caries lesions.
- The second digit codes 3 and 4 were collapsed into a single D2 code (cavitated lesion). This was done because any degree of cavitation is generally regarded as requiring intervention.

- The second digit codes 5 and 6 were collapsed into a single D3 code (cavitated lesion into dentin).

For purposes of our primary analysis, calls that require intervention are treated identically, so the D2 and D3 calls are collapsed into one category.

\section{Primary Study Outcome}

The primary study outcome is the net caries increment (root and coronal surfaces combined) from baseline (V1) to end-of-study (V6), scored as the number of changes recorded from V1 to V6, including reversals. This measure permits only one transition per tooth surface (the V5 visit is used only to achieve a more efficient multiple imputation, see below) and allows for theoretically plausible reversals (e.g., from D1 to sound) as well as for implausible reversals that presumably result from measurement error in one of the calls (e.g., D2 to sound). Three additional measures of caries increment (Table 5) will be included as secondary outcomes, to give a comprehensive view of demineralization and remineralization.

The net caries increment is computed as the sum, across tooth surfaces, of transition scores (weights) associated with 121 pre-defined transitions in tooth-surface integrity (Table 6). Our preliminary weighting scheme, as shown in the table, assigned a range of -2 to +2 , with transitions to a worse status receiving positive weights

Table 4 The PACS Taxonomy for Scoring Tooth Surfaces

\begin{tabular}{cll}
\hline $\begin{array}{c}\text { PACS } \\
\text { code }\end{array}$ & \multicolumn{1}{c}{ Definition } & Corresponding ICDAS codes \\
\hline S & sound or pits-and-fissures sealant on sound surface & $00,10,20$ \\
\hline D1 & non-cavitated lesion on otherwise sound or sealed surface & $01,02,11,12,21,22$ \\
\hline D2 & cavitated lesion on otherwise sound or sealed surface & $03,04,05,13,14,15,16,23,24,25$ \\
\hline D3 & cavitated lesion extending into the dentine on an otherwise sound or sealed surface & 06,26 \\
\hline F & filling on otherwise sound or sealed surface & $30,40,70,80$ \\
\hline FD1 & non-cavitated lesion on surface already having a filling & $31,32,41,42,71,72,81,82$ \\
\hline FD2 & cavitated lesion on surface already having a filling & $33,34,35,43,44,45,46,73,74,75,83,84$, \\
\hline FD3 & cavitated lesion extending into the dentine on a surface already having a filling & 85 \\
\hline C & full crown on surface otherwise sound or sealed & $36,76,86$ \\
\hline CD1 & non-cavitated lesion on surface already partially covered by a crown & 50,60 \\
\hline CD2 & cavitated lesion on surface already partially covered by a crown & $51,52,61,62$ \\
\hline CD3 & cavitated lesion extending into the dentine on a surface already partially covered by a & 56,66 \\
\hline crown & unscorable or invisible surface (e.g., unexposed root) & $96,54,55,63,64,65$ \\
\hline M & surface on missing tooth & 97,98 \\
\hline
\end{tabular}


Table 5 Analytical Models of Caries Increment

\begin{tabular}{ll}
\hline Model & Description \\
\hline Crude increment & One event (change) per tooth surface is counted; no reversals are counted \\
\hline Cumulative crude increment & Multiple events are possible on a tooth surface; no reversals are counted \\
\hline Net increment & One event per tooth surface is counted; reversals are included \\
\hline Cumulative net increment & Multiple events per tooth surface are possible; reversals are included \\
\hline
\end{tabular}

and transitions (reversals) to a better status receiving negative weights. Transitions that reflect no change (e. g., D1 to D1), a change to treated status (e.g., D2 to F or $\mathrm{C}$ ), or to or from an unscorable status ( $\mathrm{Y}$ or $\mathrm{M}$ ) are scored 0 and hence effectively excluded from analysis. Remineralization from D1 to $\mathrm{S}$ is considered a plausible reversal (-1), but not from D2 or D3. These tooth-surface transitions are similar to those used by Chesters et al. [28] in their fluoride-intervention study in young adolescents. Like Chesters, we recognized that reversals from cavitated lesions (bolded in Table 6) are not plausible. After completion of bootstrapping analyses of a caries increment dataset from another study (Gullion, personal communication, 2009), we concluded that the optimal way to handle unlikely or implausible transitions is to ignore them (i.e., assign a weight of zero).
Hence for our primary analysis the bolded values in Table 6 will be ignored.

\section{Other Study Measures}

PACS assessed a number of safety measures and predictors of caries increment at baseline.

\section{Medical History}

At V1, V5, and V6, participants completed a medical history questionnaire indicating whether they had ever been diagnosed or treated for a series of common medical conditions. An additional medical-eligibility questionnaire was asked at the screening visit.

\section{Oral Health History}

At V1 and V6, participants completed a brief questionnaire regarding their oral hygiene practices and dental history.

Table 6 Transition Weighting Matrix

\begin{tabular}{|c|c|c|c|c|c|c|c|c|c|c|c|c|}
\hline \multirow{2}{*}{$\begin{array}{l}\text { Baseline } \\
\text { Visit (V1) }\end{array}$} & \multicolumn{12}{|c|}{ 13-month Visit (V6) } \\
\hline & & $\mathbf{S}$ & D1 & D2/D3 & $\mathbf{F}$ & FD1 & FD2/FD3 & $C$ & CD1 & CD2/CD3 & $\mathbf{Y}$ & M \\
\hline & $\mathrm{S}$ & 0 & 1 & 2 & 2 & 2 & 2 & 0 & 0 & 0 & 0 & 0 \\
\hline & D1 & -1 & 0 & 1 & 1 & 1 & 1 & 1 & 1 & 1 & 0 & 0 \\
\hline & D2/D3 & -2 & -1 & 0 & 0 & 1 & 0 & 0 & 0 & 0 & 0 & 0 \\
\hline & $\mathbf{F}$ & -2 & -1 & 0 & 0 & 1 & 2 & 0 & 1 & 2 & 0 & 0 \\
\hline & FD1 & -2 & -2 & -1 & -1 & 0 & 1 & 1 & 1 & 1 & 0 & 0 \\
\hline & FD2/FD3 & -2 & -2 & -2 & 0 & -1 & 0 & 0 & 0 & 0 & 0 & 0 \\
\hline & $C$ & -2 & -1 & 0 & 0 & 1 & 2 & 0 & 1 & 2 & 0 & 0 \\
\hline & CD1 & -2 & -2 & -1 & -1 & 0 & 1 & -1 & 0 & 1 & 0 & 0 \\
\hline & CD2/CD3 & -2 & -2 & -2 & -2 & -1 & 0 & 0 & -1 & 0 & 0 & 0 \\
\hline & $\mathrm{Y}$ & 0 & 0 & 0 & 0 & 0 & 0 & 0 & 0 & 0 & 0 & 0 \\
\hline & $M$ & 0 & 0 & 0 & 0 & 0 & 0 & 0 & 0 & 0 & 0 & 0 \\
\hline
\end{tabular}

KEY

$\mathrm{S}=$ sound or pits-and-fissures sealant on sound surface (ICDAS codes $00,10,20$ ).

D1 = non-cavitated lesion on otherwise sound or sealed surface (ICDAS codes 01,02,11,12,21,22).

D2/D3 = cavitated lesion on otherwise sound or sealed surface (ICDAS codes 03,04,05,06,13,14,15,16,23,24,25,26).

$\mathrm{F}=$ filling on otherwise sound or sealed surface (ICDAS codes 30,40,70,80).

FD1 = non-cavitated lesion on surface already having a filling (ICDAS codes $31,32,41,42,71,72,81,82$ ).

FD2/FD3 = cavitated lesion on surface already having a filling (ICDAS codes 33, 34, 35, 36, 43, 44, 45, 46, 73, 74, 75, 76, 83, 84, 85, 86).

$C=$ full crown on surface otherwise sound or sealed (ICDAS codes 50,60).

CD1 = non-cavitated lesion on surface already partially covered by a crown (ICDAS codes $51,52,61,62$ ).

CD2/CD3 = cavitated lesion on surface already partially covered by a crown (ICDAS codes $53,54,55,56,63,64,65,66)$.

$\mathrm{Y}=$ unscorable or invisible surface (e.g., unexposed root) (ICDAS code 96,99).

$M=$ surface on missing tooth (ICDAS code 97,98).

NOTE: some of the ICDAS codes listed may be specific to children, and are included only for completeness. 


\section{Soft Tissue Examination}

Participants were examined for abnormalities of the oral mucosa at V1, V5, and V6. An assessment for candidiasis was part of this examination. This assessment also provides additional data to the microbiology substudy at the Tufts clinical center.

\section{Adverse Events}

Starting at V1, participants were asked if they had experienced any adverse events since the last visit. Additionally, at the treatment visits they were asked if they had experienced any adverse events during the treatment application. All serious adverse events (SAEs) were immediately reported to the data-coordinating center, the Project Office, the Study Chair and CHX Technologies Inc.; CHX Technologies Inc., in turn, notified the FDA. The respective Institutional Review Boards of each institution were also notified of adverse events.

\section{Medication Use}

Prescription medication use was tracked at each clinic visit.

\section{Demographic Information}

Demographic information was collected as part of the screening visit.

\section{Pregnancy Status}

Pregnant women and women who were planning to conceive during the course of the study were excluded from participation. As a safety precaution, we confirmed pregnancy status again at V5 and pregnant women did not receive the final treatment application.

\section{Acidic Drink Consumption}

The consumption of acidic beverages can prematurely erode the protective coating that was applied in stage 2 of the treatment application process. Participants were asked to avoid drinking such beverages for three days following treatment application, and we asked about typical consumption of acidic beverages at the SV, V4, $\mathrm{V} 5$, and V6 visits.

\section{Intervention and Dental Care Costs}

Program cost and cost-effectiveness analyses of the chlorhexidine coating will be assessed from patient and dental plan perspectives [30]. Study staff conducted comprehensive chart reviews to document all dental-care procedures (CDT and local codes), procedure dates, billed claims, amount paid by insurance, and insurance plan type for encounters of study participants from the randomization date to V6. Procedures and clinical service costs related to SAEs were noted. We obtained clinical staff earnings and clinic facility space to estimate provider training and service delivery costs. We also included participant travel time and costs for each clinical visit. These data will be used to calculate the incremental net cost-effectiveness per prevented caries increment of the intervention, compared to placebo, for total and restoration-related dental care expenditures. All costs will be expressed in a reference year values (e.g., 2009 dollars). Expenditures and outcomes occurring in month 13 will be discounted to present value terms using a financial discount rate for the medical-care service sector. Sensitivity analyses will be conducted by varying key model inputs, and subgroup analyses will be conducted if supported by the data. We also conducted a price opinion survey at SV to estimate patients' willingness to pay for the service and to support a cost-benefit analysis of the intervention [31]. TCRHCC participants receive free out-of-pocket care and were excluded from the price survey.

\section{Quality Control}

In addition to being trained as caries examiners and recorders, staff were centrally trained and certified in all aspects of study operations, including questionnaire administration, data entry, and application of the dental coatings. Initial training occurred prior to the start of randomization, with recertification occurring roughly annually. The data-coordinating center monitored study progress on an ongoing basis and generated regular trial monitoring reports for review by the Steering Committee and the Data Safety Monitoring Board (DSMB).

The data-coordinating center used a secure, webbased application for data entry and management. This application incorporated real-time error checking and quality assurance at the time of data entry and prompted clinical center staff about potentially erroneous data during data entry. Additional "back-end" checks were performed at the data-coordinating center.

In addition to the routine trial monitoring reports generated by the data-coordinating center, Internetenabled reporting tools allowed authorized clinical center staff to access additional reports and edit data on an ad hoc basis. The data-coordinating center maintained an electronic audit trail of all errors and error resolutions.

PACS employed three types of site visit monitoring. $\mathrm{CHX}$ Technologies Inc. conducted their own ongoing monitoring. Schiff \& Associates, an outside contractor employed by $\mathrm{CHX}$ Technologies Inc., conducted three independent monitoring visits at each clinical center-at the beginning, middle, and end of the study. And finally, the data-coordinating center conducted annual site visits at each clinical center to review all aspects of quality assurance, as defined in GCP [32].

\section{Data Analysis}

Analyses will be carried out in three samples. The intent-to-treat (ITT) sample includes all randomized participants, regardless of their adherence to the protocol, and classifies them according to their assigned treatment group. The per-protocol sample consists of participants who received all five applications of their 
assigned study treatment, or who were removed from treatment due to an adverse event, and did not deviate from the protocol in any significant way that could have affected the results. The safety sample consists of all participants who received at least one study treatment. These individuals are classified according to the actual treatment received.

In order to include all randomized participants in the ITT sample, we will use multiple imputation [33] to impute missing caries examination data for the V6 (final) visit. The planned primary- outcome analysis will be repeated in exactly the same way in each of the imputed datasets, and the results combined using Rubin's [34] rules to produce the adjusted estimates and statistics from which inferences will be drawn.

All statistical hypothesis tests will be performed with two-sided type I error level of $\alpha=0.05$. Interaction terms, when included in the model (e.g., treatment-by-site), will be assessed at $\alpha=0.10$. No adjustment for multiple testing is planned.

\section{Primary Analysis}

The primary efficacy analysis will be in the ITT population, using analysis of covariance (ANCOVA), with treatment group, clinical center, and examiner included as categorical factors, and age and age-squared as continuous covariates. We will use rank-normalized net caries increment scores in this analysis. We next will evaluate whether an interaction exists between clinical center and treatment and, if so, will estimate and test center-specific treatment effects.

\section{Secondary Analyses}

Secondary Aim 1 We will test whether the effect of the study medication differs within subgroups of the ITT sample defined by: baseline decayed filled surfaces (DFS) (median split), age (quartile groups), sex, and whether these added covariates account for any clinical center differences observed in the primary analysis.

Secondary Aim 2 We will evaluate incidence and increment of root and coronal caries in ITT participants by age, socioeconomic status (education, income), dental care setting (managed care, fee-for-service, etc.), and health-related profile.

Secondary Aim 3 We will evaluate the impact that various models of caries increment and detection criteria have on incidence and prevalence estimates of this most common adult disease.

Secondary Aim 4 We will evaluate the effect of the chlorhexidine $(10 \% \mathrm{w} / \mathrm{v})$ dental coating on resistant $(S$. mutans) and opportunistic (C. albicans) infections in the oral cavity using the subset of Tufts participants who are enrolled in the substudy.

\section{Sensitivity Analyses}

We will conduct a number of sensitivity analyses to evaluate the robustness of the primary efficacy analysis with respect to dropouts. In addition, the primary analysis and all sensitivity analyses also will be performed in the "per-protocol" sample.

\section{Safety Analyses}

Adverse events are classified using the MedDRA system [35] and categorized by system/diagnosis group, severity, relationship to study drug, action taken, and outcome. Tabulations will be prepared showing number and percent of subjects affected by an adverse event by treatment group, seriousness, relatedness, and level of cumulative exposure. The safety sample will be used for this analysis.

\section{Study Power}

The power analysis takes account of the fact that we plan to transform the observed caries-increment scores to a normal distribution. We used a data simulation to determine what the impact of the transformation would be on the target effect size of $20 \%$ reduction in net caries increment. The results of our sample-size modeling, for varying effect sizes (Cohen's $d$, i.e., the ratio of mean difference to standard deviation (SD)), are shown in Figure 1. The 20\% target effect size-expressed in rank-transformed units-is a $d$ of 0.225 , at which 416 randomized per group yields a power of $90 \%$. The final target sample size of 1000 (500 per group) was deemed sufficient to provide adequate power, given what we believe to be conservative estimates of the effect size. These calculations do not adjust for attrition since we plan to use multiple imputation to obtain plausible replacement values for missing outcome measures.

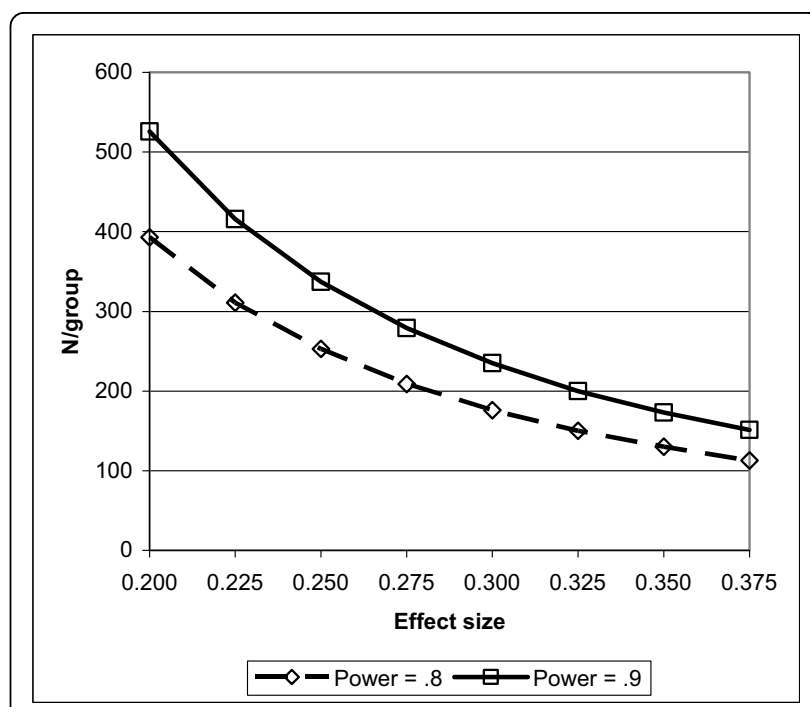

Figure 1 Sample size needed at varying effect sizes, using normalized Poisson data. 


\section{Discussion}

Adult dental caries is an expensive and chronic condition for many Americans, particularly certain minority populations. PACS will provide useful data upon which health-care policy makers and the dental community can base decisions about whether to regularly include the use of chlorhexidine coating to decrease or prevent caries in adults. Our study will also analyze whether this treatment can be used cost-effectively in a variety of settings. The latter feature is particularly important given the present state of knowledge, as few dental preventive interventions have been appraised in terms of the economic implications of their cost and their benefit.

The PACS population is highly diverse, both in terms of the participants themselves and the dental delivery systems from which they were recruited. In the study's Navajo and Hopi population in the Southwest, no outof-pocket direct payments are made by clients and indemnity is explicitly assumed by the care system; in the Pacific Northwest, the system is a not-for-profit dental Health Maintenance Organization (HMO) catering primarily to a white population that enjoys dental insurance coverage through employers; and in Massachusetts, the population is a mixed group served by a large dental insurance carrier under various service schema, as well as a study group recruited from Greater Boston by a large university dental clinic serving a primarily uninsured population. While these groups will be pooled for the primary analysis, secondary analyses will look at treatment effects in selected subgroups.

We identified several priorities in creating the PACS taxonomy for classifying dental caries. Priorities were creating the taxonomy needed to recognize the growing focus of dentistry on managing the non-cavitated lesion; being primarily visual and requiring only gentle probing to determine cavitation or the texture of the lesion base; being easy to use and reproducible; being backwardly compatible with conventional systems (e.g., Radike, World Health Organization, NIDCR); and avoiding distinguishing between active and non-active lesions to improve reliability of examiners. The resulting PACS taxonomy allows for identification of root carious lesions using a D1-D2 classification within the ICDAS descriptors, as well as allowing for identifying caries lesions associated with existing restorations for both coronal and root caries. This system is being used successfully in a separate, ongoing controlled clinical trial of the chlorhexidine coating in four American Indian communities.

Very few longitudinal studies of adult caries (1-4) have been conducted over the last 10 years. The use of D1 lesions in this trial will test the expanded visual diagnostic thresholds of ICDAS II in a large-scale randomized clinical trial. It is theorized that the use of D1 lesions to evaluate caries progression will permit shorter trials with fewer participants and better subject retention. If successful, such use will have implications for future dental research evaluating dental therapies. The PACS study is the first Phase III clinical trial to test this new model.

The need for new, cost-effective treatments to prevent or hinder the progression of adult caries is considerable. This study, together with other ongoing and completed trials of a chlorhexidine coating, is an important contribution to the establishment of sound evidence regarding the efficacy of this promising caries-prevention intervention.

\section{Acknowledgements}

Primary funding for the study was provided by the National Institute for Dental and Craniofacial Research (NIDCR). CHX Technologies Inc. provided the study treatments and some funding for: the microbiology ancillary study carried out at the Tufts clinical center, economic analyses conducted by the data coordinating center, and the calibration of dental examiners and recorders. The Project Officer from NIDCR served as a voting member on the Steering Committee and CHX Technologies Inc. participated in Steering Committee meetings as a nonvoting member.

Grant numbers: U01-DE017745-01-PHIPPS, U01-DE017746-01- SNYDER, U01DE017747-01-PAPAS, U01-DE017748-01-PAPAS, U01 DE017752 Vollmer (PI) 7/ $1 / 2006$ to $5 / 31 / 2010$ funded by the National Institute of Dental and Craniofacial Research (NIDCR). Additional financial support for the study, including provision of study treatments and limited support for some aspects of the protocol, was provided by CHX Technologies Inc.

\section{Author details}

'Center for Health Research, 3800 N. Interstate Blvd, Portland, Oregon 97227, USA. ${ }^{2}$ Tufts University School of Dental Medicine, 1 Kneeland St., Boston, MA 02111, USA. ${ }^{3}$ Dept. of Operative Dentistry CB\#7450, University of North Carolina, Chapel Hill NC 27599- 7450, USA. ${ }^{4}$ Indiana University School of Dentistry, 415 Lansing St., Indianapolis IN 46202, USA. ${ }^{5}$ Permanente Dental Associates, 500 NE Multnomah St, Suite 100, Portland OR 97232, USA. ${ }^{6}$ DentaQuest Institute, 2400 Computer Dr, Westborough MA 01581, USA.

\section{Authors' contributions}

All of the listed authors substantively contributed to the design or conduct of the study, participated in the preparation of this manuscript, and approved the final version of this manuscript. WMV, ASP, JDB, GM, CMG, JH, JSS, JLF, and BAW contributed to the study conception and design. ASP, $G M, J H, J L F, R L L$, and BAW contributed to the acquisition of data. WMV, $\mathrm{CMG}$, and $\mathrm{JH}$ analyzed and interpreted data. CMG provided statistical analysis. WMV, ASP, CMG, and RLL drafted the manuscript. ASP, JDB, GM, $C M G, J H, J S S, J L F, R L L$, and BAW critically revised the manuscript. ASP, GM, $J H, J S S, J L F$, and RLL provided administrative, technical, or material support. WMV, JH, JLF, RLL, and BAW provided supervision. WMV, ASP, JH, JLF, and BAW obtained funding.

\section{Competing interests}

The study was initially conceived by $\mathrm{CHX}$ Technologies Inc., which recruited the study investigators and actively participated in the protocol development. Although primary funding for the study came from the National Institute of Dental and Craniofacial Research (NIDCR), CHX Technologies Inc. did provide the study treatments as well as financial support for some aspects of the protocol, including full support for the planned cost analysis (funding to JLF) and limited funding to support the microbiology substudy (funding to ASP). The study will also be used by $\mathrm{CHX}$ Technologies Inc. as a "pivotal" study under US Food and Drug Administration IND \#45,466. Although CHX Technologies Inc. participated in Steering Committee discussions, it was not a voting member of the Steering Committee and is not part of any writing committees. 
Apart from the above involvement by $\mathrm{CHX}$ Technologies Inc., the authors have no financial or non-financial competing interests to declare relative to this manuscript.

Received: 28 April 2010 Accepted: 5 October 2010

Published: 5 October 2010

\section{References}

1. Griffin SO, Griffin PM, Swann JL, Zlobin N: New coronal caries in older adults: implications for prevention. J Dent Res 2005, 84:715-720.

2. Fure S: Ten-year incidence of tooth loss and dental caries in elderly Swedish individuals. Caries Res 2003, 37:462-469.

3. Broadbent JM, Thomson WM, Poulton R: Progression of dental caries and tooth loss between the third and fourth decades of life: a birth cohort study. Caries Res 2006, 40:459-465.

4. Chauncey HH, Glass RL, Alman JE: Dental caries. Principal cause of tooth extraction in a sample of US male adults. Caries Res 1989, 23:200-205.

5. US Department of Health and Human Services: Oral health in America: A report of the Surgeon General Rockville, MD: Department of Health and Human Services, National Institute of Dental and Craniofacial Research, National Institutes of Health 2000.

6. Winn DM, Brunelle JA, Selwitz RH, Kaste LM, Oldakowski RJ, Kingman A, Brown LJK: Coronal and root caries in the dentition of adults in the United States, 1988-1991. J Dent Res 1996, 75(Spec No: 642-651).

7. Centers for Disease Control and Prevention, NCHS: Vital Statistics, Series 10, Number 22, Summary of health statistics for U.S. adults: National health interview survey 2002.

8. del Aguila MA, Anderson M, Porterfield D, Robertson PB: Patterns of oral care in a Washington State dental service population. J Am Dent Assoc 2002, 133:343-351.

9. Indian Health Service: The 1999 oral health survey of American Indian and Alaska Native dental patients: Findings, regional differences and national comparisons Rockville, MD: U.S. Department of Health and Human Services 2001.

10. Greene VA: Underserved elderly issues in the United States: burdens of oral and medical health care. Dent Clin North Am 2005, 49:363-376.

11. Brown LJ, Wall TP, Manski RJ: The funding of dental services among U.S. adults aged 18 years and older: recent trends in expenditures and sources of funding. J Am Dent Assoc 2002, 133:627-635.

12. Centers for Disease Control and Prevention: Total tooth loss among persons aged $>$ or $=65$ years-selected states, 1995-1997. MMWR Morb Mortal Wkly Rep 1999, 48:206-210.

13. Reid BC, Hyman JJ, Macek MD: Race/ethnicity and untreated dental caries: The impact of material and behavioral factors. Community Dent Oral Epidemiol 2004, 32:329-336.

14. Locker D, Leake $\mathrm{J}$ : Inequities in health: Dental insurance coverage and use of dental services among older Ontario adults. Can J Public Health 1993, 84:139-140.

15. Millar WJ, Locker D: Dental insurance and use of dental services. Health Rep 1999, 11:55-67.

16. $\mathrm{NIH}$ : Diagnosis and management of dental caries throughout life. $\mathrm{NIH}$ Consensus Statement 2001, 18:1-23.

17. Bader JD, Shugars DA, White BA, Rindal DB: Development of effectiveness of care and use of services measures for dental care plans. J Public Health Dent 1999, 59:142-149.

18. Bader JD, Shugars DA, White BA, Rindal DB: Evaluation of audit-based performance measures for dental care plans. J Public Health Dent 1999, 59:150-157.

19. Baysan A, Lynch E, Ellwood R, Davies R, Petersson L, Borsboom P: Reversal of primary root caries using dentifrices containing 5,000 and $1,100 \mathrm{ppm}$ fluoride. Caries Res 2001, 35:41-46.

20. Wyatt CC, Maupome G, Hujoel PP, MacEntee MI, Persson GR, Persson RE, Kiyak HA: Chlorhexidine and preservation of sound tooth structure in older adults. A placebo-controlled trial. Caries Res 2007, 41:93-101.

21. Banting DW, Papas A, Clark DC, Proskin HM, Schultz M, Perry R: The effectiveness of $10 \%$ chlorhexidine varnish treatment on dental caries incidence in adults with dry mouth. Gerodontology 2000, 17:67-76.

22. Forgie AH, Paterson M, Pine CM, Pitts NB, Nugent ZJ: A randomised controlled trial of the caries-preventive efficacy of a chlorhexidinecontaining varnish in high-caries-risk adolescents. Caries Res 2000, 34:432-439.
23. Code of Federal Regulations. 21 CFR 210, 21 CFR 211. 2009.

24. Health Canada Drug Identification Number 02046245. 2009.

25. Irish Medicines Board product license PA1205/1/1. 2009.

26. Pitts NB, Fyffe HE: The effect of varying diagnostic thresholds upon clinical caries data for a low prevalence group. J Dent Res 1988, 67:592-596.

27. Papas A, Russell D, Singh M, Kent R, Triol C, Winston A: Caries clinical trial of a remineralising toothpaste in radiation patients. Gerodontology 2008, 25:76-88.

28. Chesters RK, Pitts NB, Matuliene G, Kvedariene A, Huntington E, Bendinskaite R, Balciuniene I, Matheson JR, Nicholson JA, Gendvilyte A, Sabalaite R, Ramanauskiene J, Savage D, Milerine J: An abbreviated caries clinical trial design validated over 24 months. J Dent Res 2002, 81:637-640.

29. ICDAS II. 2009 [http://www.icdas.org/].

30. Haddix AC, Teutsch SM, Corso PS: Prevention effectiveness: A guide to decision analysis and economic evaluation New York: Oxford University Press, 22003.

31. Gold M, Siegel J, Russel L, Weinstein M: Cost effectiveness in health and medicine New York: Oxford University Press 1996.

32. International Conference on Harmonisations, Guidance for Industry. E6 Good Clinical Practice: Consolidated Guidance; 1996 [http://www.fda. gov/downloads/drugs/guidancecomplianceregulatoryinformation/ guidances/ucm073122.pdf], 1-30-2009.

33. Schafer JL, Graham JW: Missing data: our view of the state of the art. Psychol Methods 2002, 7:147-177.

34. Rubin DB: Multiple imputation of nonresponse in surveys New York: John Wiley \& Sons, Inc. 1987.

35. MedDRA Maintenance and Support Services Organization. MedDRA the Medical Dictionary for Regulatory Activities. 2005 [http://www. meddramsso.com/index.asp], International Federation of Pharmaceutical Manufacturers and Associations (IFPMA).

\section{Pre-publication history}

The pre-publication history for this paper can be accessed here: http://www.biomedcentral.com/1472-6831/10/23/prepub

\section{doi:10.1186/1472-6831-10-23}

Cite this article as: Vollmer et al.: Design of the Prevention of Adult Caries Study (PACS): A randomized clinical trial assessing the effect of a chlorhexidine dental coating for the prevention of adult caries. BMC Oral Health 2010 10:23.

\section{Submit your next manuscript to BioMed Central and take full advantage of:}

- Convenient online submission

- Thorough peer review

- No space constraints or color figure charges

- Immediate publication on acceptance

- Inclusion in PubMed, CAS, Scopus and Google Scholar

- Research which is freely available for redistribution

Submit your manuscript at www.biomedcentral.com/submit
C Biomed Central 